\title{
Dynamic Shifts in Visual Focus of Eye Movements Emphasize Associative Relevancy
}

\author{
Gufran Ahmad \\ ${ }^{1}$ College of Computer Sciences and Information Systems, Jazan University, Saudi Arabia \\ Correspondence: Gufran Ahmad, College of Computer Sciences and Information Systems, New Campus, Jazan \\ University, Jazan, 45142, Saudi Arabia. Tel: 966-503-082-048.
}

Received: October 17, 2015

Accepted: November 5, 2015

Online Published: November 27, 2015

doi:10.5430/rwe.v6n4p18

URL: http://dx.doi.org/10.5430/rwe.v6n4p18

\begin{abstract}
Progress in the studies of eye movements have touched an innovative milestone and are steadily pushing forward brand-new interdisciplinary researches. In a number of common to complex scenarios, the applicabilities of eye tracking guarantee absolute success in the understanding of human behaviors and interactions. The eye movement probes result in understanding of the underlying mechanism of visual perception and dynamics of humans' cognition that are of prime concerns for a number of social, economic, and scientific purposes. In this study, we conducted a series of eye tracking experiments to verify our hypothesis that during artistic portrait viewing, the visual focus of eye movements dynamically associated relevant constituents of artistic portrait. We collected the eye movement data of participants who regarded artistic portraits during active viewing. The paths produced from eye tracking system during portrait viewing traced connected focuses of eye movements in eye movements based on associative relevancy in visual contexts. These experimental facts validated the hypothesis that dynamic shifts in visual focus of eye movements emphasized associative relevancy.
\end{abstract}

Keywords: associative relevancy, cognition, eye movements, visual focus

\section{Introduction}

\subsection{Introduction and Importance}

Eye movements is one of the research studies which have prospered rapidly due to immense demands and interests of interdisciplinary researches, like human and developmental psychology, psycholinguistic and readings, neuroscience, vision research, usability studies, business marketing and advertising research, ophthalmology, human computer interaction, etc. Additionally, these studies have bettered research comprehensions, advanced tools and techniques, and widespread applications. Tracking of eye movements brings about remarkable understandings of human mind, in terms of human views and intents that are the key interests for businesses and researches. By doing so, businesses can familiarize with human behaviors and develop business strategies gradually. Indeed, businesses need people's views and intents for better understanding of market behavior that heavily relies on behavior of consumers. During a recent period of time, a number of online and offline businesses, like marketing, advertisement, shopping, search engine optimization, and web designing are transforming and reforming their business policies and targets in accordance with the novel findings of eye movements for business expansions (Bojko, 2013; Granka, Joachims, \& Gay, 2004; Hermansen, 2015; Koller, Salzberger, Brenner, \& Walla, 2012; Pan B. , et al., 2004; Pan B. , et al., 2007).

The biological structure of human eyes constitutes visual perception and remains as an integrated part of human visual system. In fact, eyes are one of the biological sensors linked to the human brain for complex mechanism of neurocognition. Additionally, the eyes undertake inseparable participation along with human brain in attentional and visual processing of information for comprehension of surrounding objects. In fact, this complex mechanism of neurocognition is a combination of numerous underlying processes inhabiting in human mind. The human mind incites and causes cognitive processes, like human's sensation, consciousness, visual attention, perception, meta-cognition, reasoning, analogical thoughts, information processing, and other concerned processes (Brown, 1999; Duchowski, 2003; Wells, 2000; Tommasi, Peterson, \& Nadel, 2009; Ahmad, 2014; Ahmad, 2015).

In case of active viewing, the eyes move visual focus to capture sight of a specific section of the visible area as the 
human mind has tendency to comprehend the degree of detail visible in the central direction of visual focus of eye movements. During the process of eye movements, eyes pass through two temporal phases: fixations (the stops or periods of time when point of focus or significant look is relatively slow) and saccades (the hops between stopping points). Saccades are often information seeking and directed to specific visual objects or areas by the requirements of ongoing behavior. This implies the existence of cognitive processes of eye movements in viewing as well. Therefore, the underlying mechanism of visual viewing is sequential and coordinated phenomena of cognitive as well as correlated processes (Duchowski, 2003; Gompel, Fischer, Murray, \& Hill, 2007; Henderson \& Hollingworth, 1998; Hoffman, 1998; Holsanova, 2008; Snowden, Thompson, \& Troscianko, 2012; Stark \& Ellis, 1981; Tsotsos, 2011; Ahmad, 2014; Ahmad, 2015).

\subsection{Relevant Background and Research}

The study of relationships of fixation sequences of eye movements to the behavior of usual human activities has its origin. In fact, inherent salience of objects is not accountable for focal shifts in eye movements, but by their importance to the task or context in hand. In contradiction of free viewing, the movements of eyes and contextual acts are associative in nature and have a chain of linking. Moreover, contextual acts compose of a number of perspectives including an act of associativity within the contexts, elements, or intents. Such associativity has built upon the sense of relevancy among the contexts of the object (Bly \& Rumelhart, 1999; Duchowski, 2003; Gompel, Fischer, Murray, \& Hill, 2007; Griffin, 2004; Henderson \& Hollingworth, 1998; Henderson \& Hollingworth, 1999; Holsanova, 2008; Hoffman, 1998; Liversedge, Gilchrist, \& Everling, 2011; Stark \& Ellis, 1981; Ahmad, 2015).

In addition, a focused visual appearance, i.e. spotlight metaphor, enables and constructs the visual focus in eye movements during artistic portrait viewing. The center of focused attention in visual area is considered as having more brightness than areas to which attention is not focused or areas from which attention has been detached. The spotlight of eye movements switches off at one location and then switches on at other. Besides, as human's capabilities for attention and processing for information has restraints cognitively, so it is rather challenging to consider about focusing everything at a time. Cognitively, human gives attention to small part of visual object, one at a time and likewise, human can focus on only small piece of information at a time. This is why the human processing of information is reasonably gradual as it works on the mechanism of human cognition (Bojko, 2013; Duchowski, 2003; Gompel, Fischer, Murray, \& Hill, 2007; Henderson \& Hollingworth, 1998; Hoffman, 1998; Holsanova, 2008; Rayner, 1992; Ahmad, 2014; Ahmad, 2015).

As a further matter, there occur numerous impact factors, which engender reasoning, notion of analogy, flow of thoughts, meta-cognition, and other pertinent cognitive processes during the eye movements. We regard in a location of portrait that is partly determined by the portrait's constraints and region's informative description, partly by the task, intent, context, or interest. Viewers can arrange diverse visual paths through the same portrait, since they extract information from those parts of the portrait to describe particularly. Therefore, the evolved flow of thoughts cognitively, motivates the conscious focus of eye movements to change to the next contextual part of the portrait. By doing so, these impacting factors propel human visual focus of eye movements dynamically in the phenomena of eye movements (Bly \& Rumelhart, 1999; Duchowski, 2003; Gompel, Fischer, Murray, \& Hill, 2007; Griffin, 2004; Henderson \& Hollingworth, 1998; Henderson \& Hollingworth, 1999; Hoffman, 1998; Holsanova, 2008; Liversedge, Gilchrist, \& Everling, 2011; Rayner, 1992; Snowden, Thompson, \& Troscianko, 2012; Stark \& Ellis, 1981; Tsotsos, 2011).

Often, artistic portraits are specimens of artworks created by human beings. In fact, art is a wide-ranging of human activities and the creations of those activities with human understandings and imaginations that involve human cognition. By looking at a piece of artwork, a fascinating sequence of neurological, perceptual, and cognitive phenomena surfaces. These phenomena direct us towards our understandability and scholarly competence about the piece of artwork instantaneously. In addition, as science is laying its foundation to understand our knowledge about art, likewise, the art offers us a view of mind that understands the art. Undoubtedly, we identify that all types of art are one of the fabulous representations in our lifetimes. It can calm down our distressed heart and motivate our mental states and spirits. Further, artistic perceptions stimulate profound thoughts as well as all types of sensations. Moreover, an art is a productive activity that focuses on the thoughtful modification and augmentation of worldviews. As a rule, all known pieces of art are creative, constructive, and metacognitive as per their roles because of being explicable and comprehensible. The types and styles of art are technology-driven as pioneering technologies convey regeneration to the artworks. The most important part of an art is its aim to put in an appearance for conscious and responsive about the art itself, and synchronously, spreading consecutive cognitive processes in human mind, so that a novel lookout may be intuited and perceived by the onlookers of artworks (Solso, 1994; Solso, 2003; Turner, 2006; 
Viegas \& Wattenberg, 2007; Ahmad, 2015).

The processes of eye movements bring about and propagate a series of streaming thoughts successively to obtain information about the portrait or object of interest. These streams of thoughts predominantly originate the notion of analogy. Although there are a number of standpoints about analogy itself, yet analogical impression is constantly accessible in visual perceptions. Based on existing research works, it gives the consideration that the standpoint of mapping and the standpoint of higher level of perception are two dissimilar looks of the same thing, i.e., analogy. Analogy is one of the inherent concepts that persist during the phenomena of eye movements (Gentner, 1983; Gentner \& Markman, 1997; Gentner \& Medina, 1998; Gentner, Rattermann, \& Forbus, 1993; Gompel, Fischer, Murray, \& Hill, 2007; Henderson \& Hollingworth, 1999; Hofstadter \& Sander, 2013; Holsanova, 2008; Tsotsos, 2011).

The process of associative relevancy initiates during the shifts in visual focus as the movements of eyes establish associations among analogical contexts or portions of interest during the active portrait viewing. Such establishments that appear in the artistic portraits or scenario maps are highly significant and known as patterns of associative relevancy. The associative relevancy patterns are observable and reflect the relict nature of in-between primordial human cognition during visual perception. In fact, associative relevancy is developmental and interrelated notion, which originates from thoughts of analogy during the process of human viewing. The influence of associative relevancy predominantly remains during cognitive phenomena of eye movements. It is an underlying mechanism for creativity and annihilation of complexity during information processing and information retrieval for better interpretation of the object of interests. Besides, it resembles as an associative chain that links contexts, intents, portions, elements, shapes, sizes, colors, contrasts, or relations based on similarity or sameness. Further, associative relevancy manipulates and generates an incitement cognitively to associate relative contexts, intents, elements, etc. (Gentner, 1983; Gentner \& Markman, 1997; Gentner \& Medina, 1998; Gentner, Rattermann, \& Forbus, 1993; Gompel, Fischer, Murray, \& Hill, 2007; Henderson \& Hollingworth, 1999; Hoffman, 1998; Hofstadter \& Sander, 2013; Holsanova, 2008; Liversedge, Gilchrist, \& Everling, 2011; Ahmad, 2015).

\subsection{Hypothesis and Corresponding Research Design}

Generally, the viewer changes visual focus of eye movements without restriction during the observations of artistic portraits, i.e. the eyes can shift visual focus from one fixation to another fixation generously. By plotting viewer's eye movements, we get a pattern of eye tracks consisting of saccades and fixations or visual focuses of eyes. The key intention of this study in artistic portrait viewing is to discover associative relevancy in the movements of eyes' visual focus and to visualize the associated relevancy patterns of eye movements caused by the shifts of visual focus of eye movements.

We make a hypothesis that dynamic shifts in visual focus of eye movements emphasize associative relevancy during the artistic portrait viewing. For the purpose, we research the visual focusing of eye movements from cognitive perspective, including the associative relevancy in the changes of visual focus during portrait viewing and analyze the patterns of sequenced visual focusing of eyes to visualize the information. Here, tracking of eye movements, comparing, and visualization of sequenced focusing patterns to extract information for interpretations are required stages of this study. By following along these stages in a number of analysis, we aim to settle the visual focus of eye movements and the nature of associativity to which eye movements belong.

\section{Method}

\subsection{Eye Tracking System}

In eye tracking system, the system illuminates infrared light for tracking the eye movements. The camera, connected to the system, captures the location of viewer's eyes in terms of fixation during experimentation time. As the viewer moves eyes to look for a new location of the scene, the camera records new fixation also. This process of recording continues subsequently. The system generates eye movement tracks and heat maps using the captured data. We utilize these data for further analysis. 
Figure 1 shows the schematic diagram of eye tracking system and basic processes involved during eye tracking experimentation.

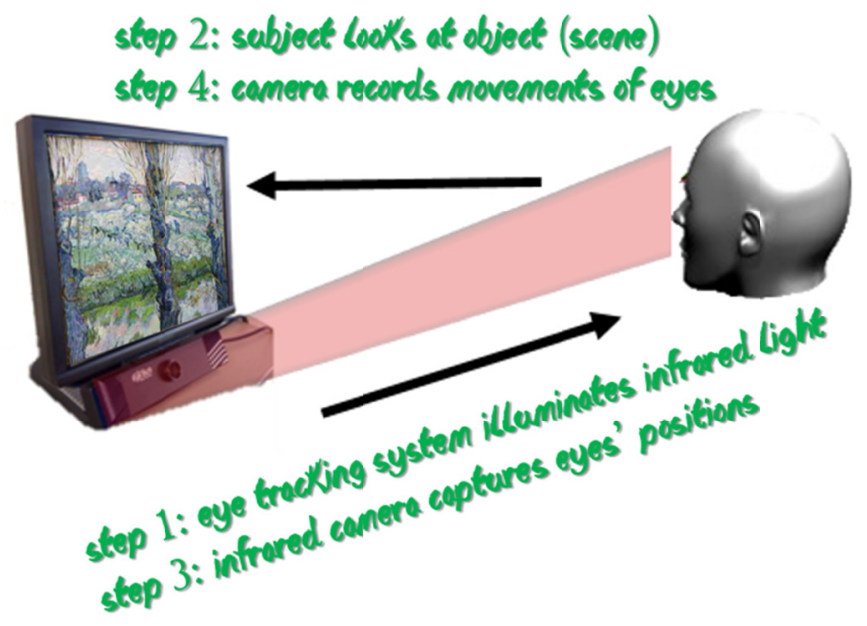

Figure 1. Eye tracking system with operational processes

We take the traces of eye movements in diverse layouts as per analyst's suitability. Among them, there are two most common formats are Heat Map and Sequenced Gazing (Visual focus) with circle of concentration. In Heat Map, we record the track of eye as illumination and intensity of infrared light rays. This is Energy Therapy Technique (ETT) based process. In Sequenced Gazing, the system assigns the eye tracks as numbered circles with their areas indicating the time duration of eye gazing in those areas respectively (Bojko, 2013; Duchowski, 2003).

In our experiments, we study the sequenced visual focusing of viewer's eye movements. The system generates sequenced focusing of viewer's eye movements during scene viewing.

\subsection{Flow Chart of Study}

The study on eye movements during portrait viewing consists of a number of footsteps. We complete these footsteps in sequence to accomplish our study. The flow chart in the below figure (Figure 2) demonstrations these footsteps. This is a comparative study of two items; one item is artistic portrait and other item is the eye movement tracks of the same portrait generated from eye tracking system.

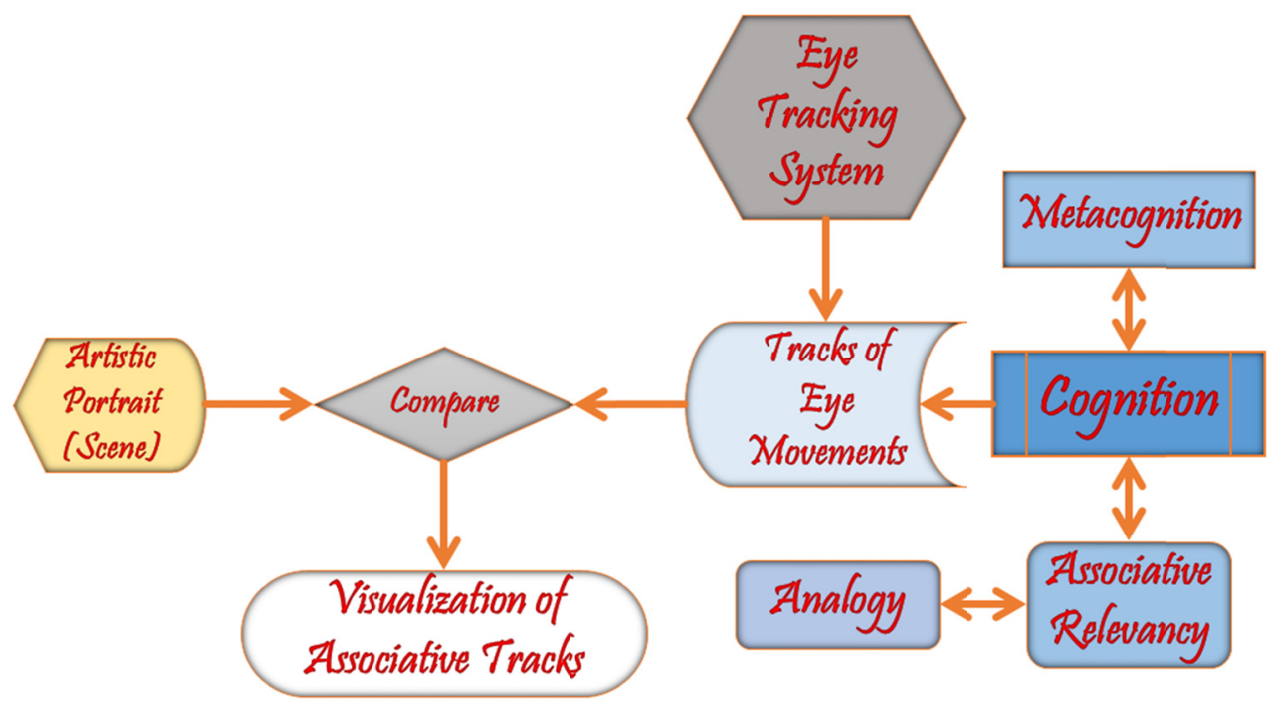

Figure 2. Flow chart of research study 
The whole procedure starts with the recording of eye movement tracks for a Subject (viewer) on eye tracking system for an Object (artistic portrait). The generated tracks of eye movement of the same artistic portrait are comparable to the original artistic portrait. This comparative analysis extrapolates visualization and interpretation of the consequence. Hence, these two items get a comparison side by side.

During comparison stage, we make use of cognitive process, and metacognitive process, in addition to associative relevancy perspective of analogy, to understand the hidden mechanism that creates resultant associative tracks of eye movements. By examining these facts along with cognitive consideration, we come up with final clarifications on these surfacing and persisting phenomenal happenings.

\subsection{Procedure}

We selected 65 participants from a number of fields within university, aging from 20 years to 41 years. These Subjects (participants) watched three purposely chosen famous artistic portraits by Vincent Van Gogh as shown below in Figure 3.

The artistic portraits were 'Fishing in spring', 'The Poet's Garden', and 'The Yellow House' by Vincent Van Gogh.
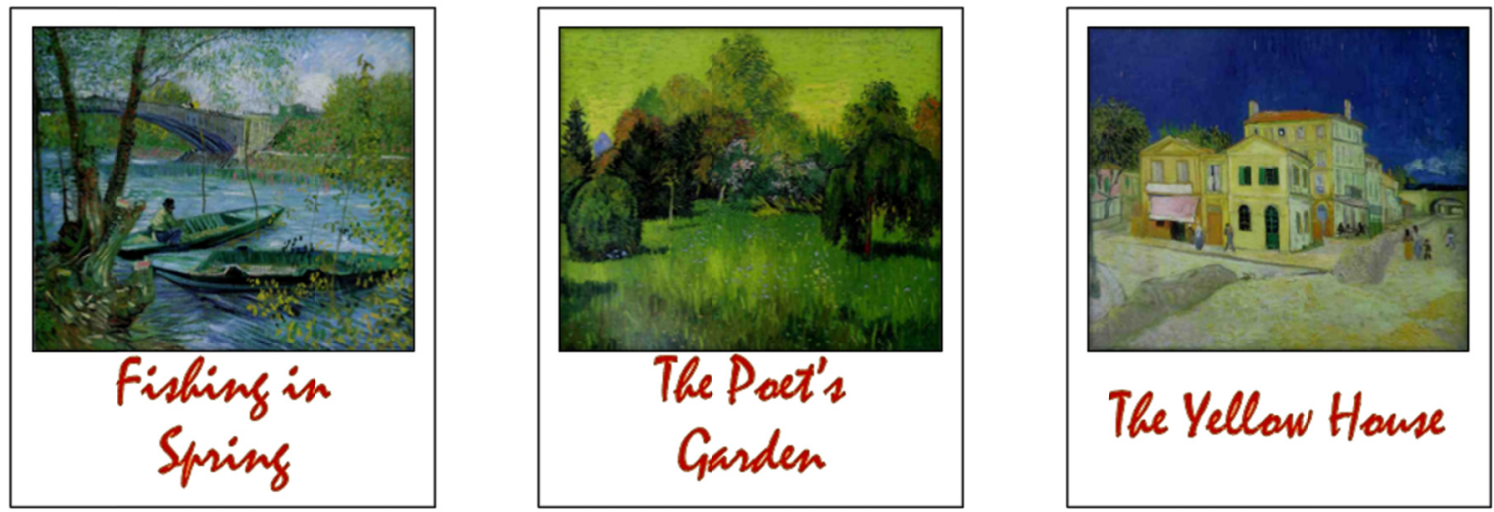

Figure 3. Selected artistic portraits for research study

We closely monitored Subjects' eye movements as they viewed 32-bits full-color artistic portraits. A computer monitor displayed the Objects (the artistic portraits) one after the other. The shown portraits were at a resolution of $1280 \times 1024$ pixels, subtended 15 degree horizontally by 10 degree vertically. Besides, the viewers regarded at the artistic portraits at a viewing distance of $75 \mathrm{~cm}$. Eye position was sampled from an Eye Tech Digital Systems TM3 $16 \mathrm{~mm}$ Eye Tracker, and eye tracking data was parsed into fixations (circles with focused time-period in areas) and saccades (sequenced focuses with linear edges).

Furthermore, we held the Subject's head steady in advance prior to experimentations. Prior to every trial, Subjects completed a procedure to calibrate the output of the eye tracker against spatial positions on the display screen. We repeated this procedure regularly throughout the experiment to maintain high level of accuracy. Subjects looked at the artistic portraits freely and generously.

Subjects observed the artistic portrait for a short duration of 25 seconds to limit perceivable attentiveness. During this time-span, the Subjects viewed the artistic portraits with their normal eyes and focused visual attention on the Object (the artistic portrait).

\section{Analysis}

During the phase of analysis, we analyzed all three portraits respectively with the purpose to expound our findings methodically in the most generalized perspective. 


\subsection{Study of Artistic Portrait 'Fishing in Spring'}

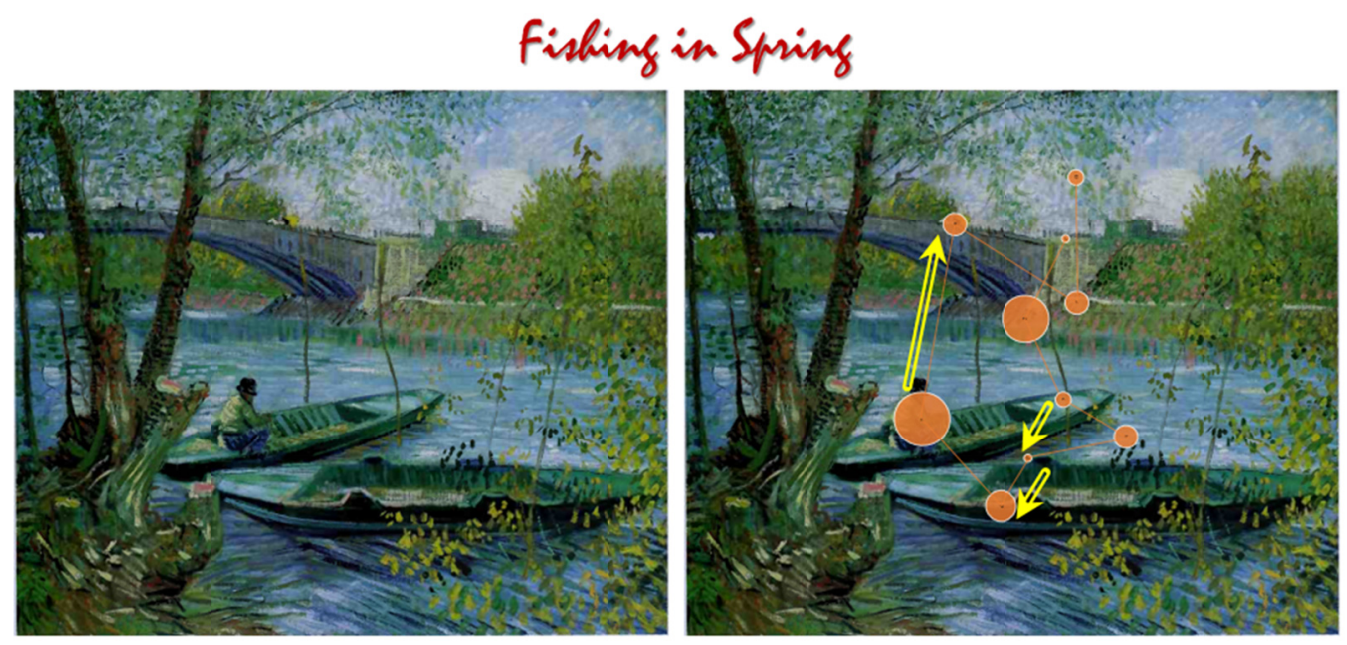

Figure 4. Portrait of 'Fishing in Spring' and sequenced visual focuses of the same portrait

In these eye-tracking experiments of Figure 4, the Subject attentively looked at artistic portrait of "Fishing in spring" having broadly spread lake, fishing man sitting on a boat and a nearby empty boat, along with a large bridge for travelers, etc. In the beginning, the Subject's sensation brought forward human consciousness to direct them gradually towards the central portions of the portrait. Here, the Subject shifted visual focus in top-down scan for information seeking saccades. After a longer pause at these locations, the Subject's visual focus located the first boat and in subsequent shifts located the second boat due to the happening of cognitive mechanism of human mind consisting of analogical thoughts along with the process of associative relevancy among the objects of interests during active viewing. The emerging phenomena of associative relevancy that linked the two contextual fields (boats) of the portrait had highlighted the association in visual focus of eye movements.

Next, the Subject's visual focus of eye movements shifted on the man sitting on the boat. At this stage, the Subject perceived knowledge about the field of the portrait, which helped them to move to the next step. In the next move of saccade, the Subject shifted visual focus on the people travelling over the bridge. Moreover, due to the existence of cognitive process during the shifts in visual focus of eye movements, the Subject sensed the flows of analogical thoughts and started to realize the idea of analogy. These cognitive phenomena compelled the Subject to associate contextual portions (humans) of the portrait due to existing relevancy among them in terms of being the objects of similarity. This evolved thought and association of relevancy continued as visual focus of eye movements shifted towards the upper portion of the portrait.

Within these stages, the visual focus of eye movements came across cognitively generated incentives that evolved flows of analogical thoughts. These thoughts derived a sense of associativity among the focused humans in subsequent movements of eyes. These types of associative chaining among relevant elements (humans) in the artistic portrait arose from cognitively induced factor and analogical thoughts. In other words, the visual focus of eye movements consistently linked the similarity in contextual structures or shapes and established the association among these objects of interest during the eye movements.

Later visual focuses of eye movements could not associate this relevancy because of the nonexistence of the any further object of interest (human) within the contextual fields. Therefore, the visual focus of eye movements randomly propagated towards a number of other fields to scan arbitrarily for visual information processing and seeking information.

Later conversations to the Subjects suggested that the visual fields of lake, and boats were the objects of interest in the beginning. Subsequently, as the Subjects viewed the other portions of the artistic portrait, the existence of humans became a matter of interest to the Subjects, as these objects were delightful and exciting things of the artistic portrait that Subjects started to pay attention. The related fields of similarity bound them to shift their visual focuses of eye movements dynamically. Hence, the conclusion came from undergoing cognitive processes of Subjects' minds 
in artistic portrait viewing that the dynamic shifts in visual focus of eye movements emphasized associative relevancy. In other word, we got definite confirmation in this analysis about our hypotheses.

\subsection{Study of Artistic Portrait 'The Poet's Garden'}

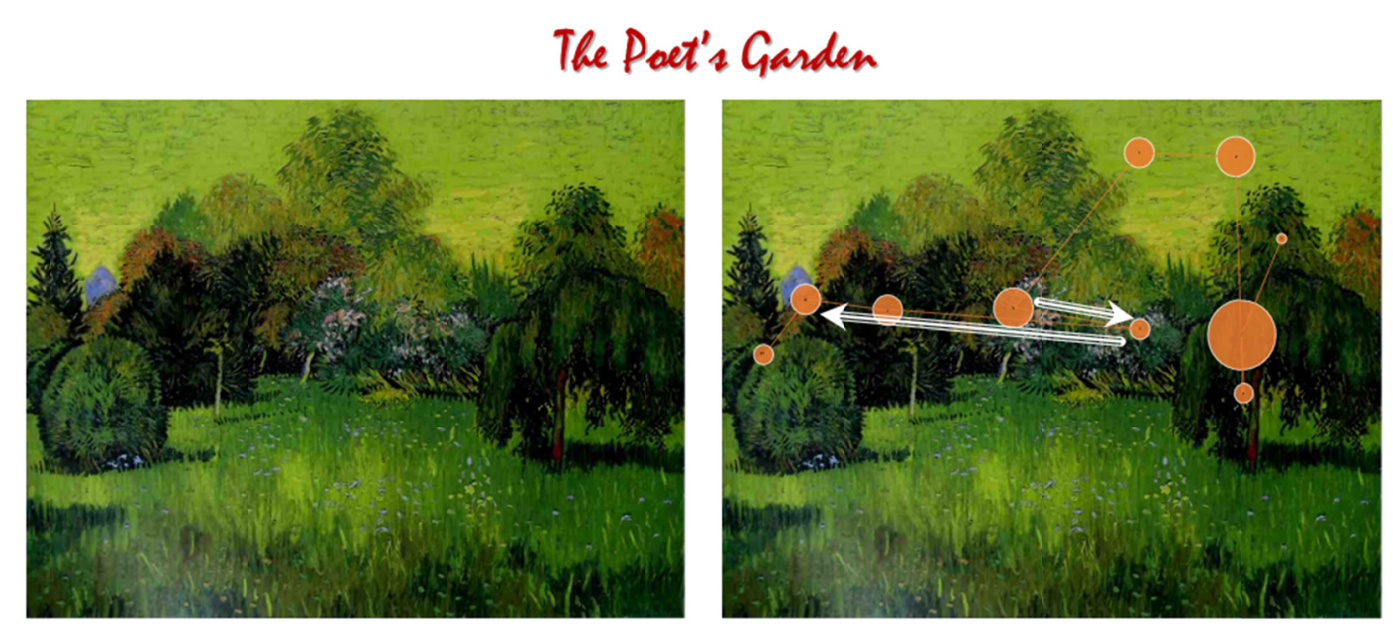

Figure 5. Portrait of 'The Poet's Garden' and sequenced visual focuses of the same portrait

In these eye-tracking experiments of Figure 5, we displayed an artistic portrait "The Poet's Garden" which was an artistic expression of a garden with a weeping tree, grass, round clipped cedar shrubs and an oleander bush. In the beginning, the consciousness of the Subject developed visual attention and the Subject started focusing at central right side (dark green color of tree) of the portrait due to brightness contrast of the field. Subsequently, visual focus of eye movements shifted towards the upper portion (intense green color of grass) of the portrait due to brightness contrast of the field again. However, these shifts sensed two opposite color contrasts to the adjacent fields of the portrait respectively.

Next, the visual focus of eye movements moved and persisted at oleander bush for processing information to perceive and retrieve the knowledge. At this stage, the Subject attained a definite flow of thought based on contextual resemblance and color contrast (whitish purple) biasing of the oleander bush. Therefore, in the following moves, the focus of visual attention relocated to the adjacent analogous fields of the portrait. This fetched visual perception to the Subjects for the knowledge of context and relevant fields. The existing cognitive process in the human minds enlightened the other coexisting processes, i.e., the process of analogical considerations and the process of linking visual elements of portrait based on associative relevancy.

The process of analogical considerations engaged the Subject's visual focus of eye movements to think about the associative contexts (oleander bush) of visual fields in the portrait. Such associative relevancy under the basis of color contrast and Object's shape originated cognitively generated incentives that exerted drive to assist visual focus of eye movements to proceed further in the direction of associatively relevant elements of the portrait. Further, the shifts in visual focus of eye movements during eye movements reflected the associative relevancy that actively linked such relevancy under the influential cognitive processes.

Later interviews of the Subjects figured out these details as well. They reported that they were actively looking at vibrant and charming oleander bush regions of the artistic portrait, as these objects in the artistic portrait were interesting to them. This established our hypotheses that the dynamic shifts in visual focus of eye movements emphasized associative relevancy during the artistic portrait viewing. 


\subsection{Study of Artistic Portrait 'The Yellow House'}

\section{The Yellow House}

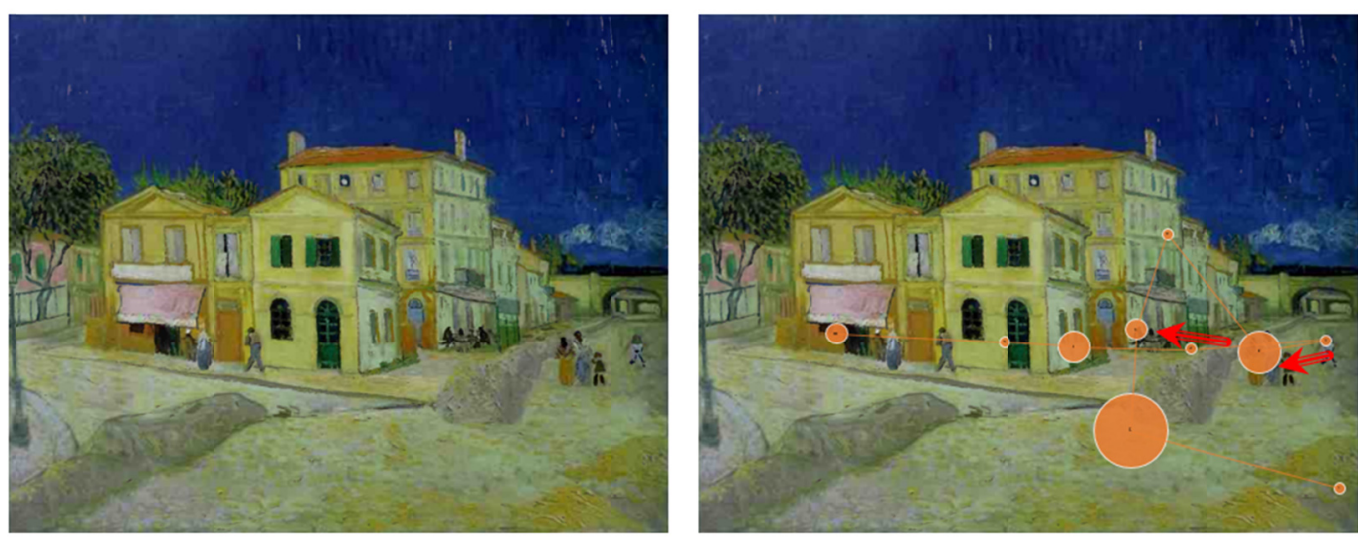

Figure 6. Portrait of 'The Yellow House' and the sequenced visual focuses of the same portrait

In these eye-tracking experiments of Figure 6, we showed an artistic portrait "The Yellow House" in which there were a number of houses, specially the yellow house rented by Van Gogh in the center of artistic portrait. The gloomy blue sky had color contrast biasing with the shiny land background of the artistic portrait under the sunshine. On the right side of the artistic portrait, people were travelling, and sitting under a shading. On the right side, a few people were walking on Pedestrian Street. In the beginning, Subject's consciousness brought visual attention on the left side of the portrait and traversed towards the right side along the central yellow house focusing on shutters and windows of the central yellow house.

Gradually, the visual focus of eye movements shifted towards the right side of the portrait as scanpath traversal across the portrait for information seeking purpose. As the visual focus came across far right at the people travelling on the road, this became the objects of interest for the Subject. The Subject cognitively initiated information retrieval from that visual field of the portrait. Hence, the Subject came across the phase of visual perception from the element of the portrait and successfully perceived. In subsequent moves, the Subject changed the visual focus of eye movements to the contiguous travelling people. At this instant, the contextual fields of the portrait revealed consideration of analogy and underlying mechanism of linking based on associative relevancy among these elements of the portrait based on their resemblances. Such associatively pertinent fields of the portrait activated cognitively controlled incentives that applied impulse on the visual focus of eye movements to shift towards the next field of relevant interest. Consequently, the visual focus of eye movements in eye movements shifted to nearby sitting people also.

Later on, the interviews of the Subjects disclosed their interest in looking for fascinating fields of people in the artistic portrait during the active viewing. Here again, our analysis determined and demonstrated our hypotheses that dynamic shifts in visual focus of eye movements emphasized associative relevancy during the artistic portrait viewing.

\section{Discussion}

In this study, the artistic portraits echo the key point of artistic creations in the form of human interactions with the art as viewing art can engage human cognitive mechanisms as a part of human interactions. Human interactions have the capabilities to let us comprehend the visual insights during artistic portrait viewing. These inspired pieces of art demonstrate intrinsic human interactions to perceive information and interpretation of realistic world in human mind for interpretation. These cognitively shaped emotional perspectives are too challenging to encapsulate from data computing and machine based analytics. Accordingly, eye movements establish and encounter these cognitive perspectives and human thoughts for associative relevancy notion in scene viewing (Viegas \& Wattenberg, 2007; Turner, 2006; Solso, 2003; Solso, 1994; Snowden, Thompson, \& Troscianko, 2012; Holsanova, 2008).

The visual focuses of eye movements shifts during artistic portrait viewing are intense and reflective stages for appropriate repossession of task-relevant visual information. Further, these are the requirements for visualization of final maps generated by eye tracking system. In this study, we notice that the generated eye movement tracks of 
sequenced visual focuses of eye movements are outstandingly associative in nature. These are most important confirmations to validate an association of analogical mapping based on contexts.

Nonetheless, the existence of time-based deviations within the traces of eye movements during artistic portrait viewing is cognitive ambiguities possibly under the occurrence of numerous flows of thoughts and unrestrained movements of eyes. We can distinguish these occurrences in other viewpoints as well. Another viewpoint about these alterations is a short-term breakdown of these associative chaining processes of analogical contexts in the above-mentioned analyses due to cognitive dissonance caused by two unrelated cognitions within a moment. Even so, the happening of associative relevancy based chaining among contexts is sure and certain proof. Further, it is unreasonable to combine the complete scenario of human cognition in the sequential eye focusing of eye movement tracks without the aspect of associative relevancy concept. Furthermore, the derivation of associative relevancy based incentive is indivisible artifact that has surfaced its emblem in the experimentations for eye movements and in turn, by the application of this incentive, the visual focus of eye movements actively associates relevancy in eye movements as well (Viegas \& Wattenberg, 2007; Turner, 2006; Tsotsos, 2011; Stark \& Ellis, 1981; Solso, 2003; Solso, 1994; Gentner, Rattermann, \& Forbus, 1993; Gentner \& Medina, 1998; Gentner \& Markman, 1997; Gentner, 1983).

Preferring an art as an assistance to be familiar with triggering processes of human cognition is a subject of discussion as well. As a human mind functions to facilitate between the environment and the necessities of the body, an artistic innovation extends engagement to improve consciousness of human mind. Further, various enhanced statuses of consciousness might be attained through art, as some experimental methods have already commenced to perform. As per existing literatures, it is unquestionable that viewing an artwork can nurture and cultivate human consciousness drastically and extensively (Solso, 1994; Solso, 2003; Turner, 2006; Viegas \& Wattenberg, 2007).

Additionally, the reclamation of associative chaining from its diverged tracks due to momentary loss of sight or visual color based cognitive impacts, in addition to the thought related to cognitive dissonance during eye movements for visual focus of eye movements shifts is noteworthy from the viewpoint of its competence. This competence of reclamation is a satisfied indication of coherent and consistent associative mapping established in between the relevant visual fields of the artistic portrait passing through human cognitive processes. These reclamations of associative relevancy are capable of gaining unique and unchanged overall consequences despite unpredicted hindrance during artistic portrait viewing.

For the period of final phases of experimentations, the visualization of associative relevancy of visual contexts in terms of associative eye movement tracks is a tactical and decisive involvement of entire activities. The visualization of associative eye movement tracks, in terms of associatively relevant visual contexts discovered in eye movements is in fact, innovative point of view for the experts who research them for specialized objectives. Even though the existence of analogical thought and associativity in relevant visual contexts or intents are accessible in the concerned literatures, yet its materialization and explication differs significantly as being separate standpoints of the same thing. In this respect, the visualization of associative eye movement tracks discloses once more the presence of associative relevancy in visual contexts or visual elements of artistic portrait during scene viewing (Viegas \& Wattenberg, 2007; Turner, 2006; Tsotsos, 2011; Stark \& Ellis, 1981; Solso, 2003; Solso, 1994; Snowden, Thompson, \& Troscianko, 2012; Rayner, 1992; Holsanova, 2008; Henderson \& Hollingworth, 1999).

Furthermore, the experimental confirmation of associative relevancy during active portrait viewing supports our hypothesis for which we performed a series of experimentations. The hypothesis that the visual focus of eye movements actively associates relevancy in eye movements is trustworthy, substantial, and groundbreaking vision related to eye movements research.

Recent trends in businesses for numerous applications of eye movements have explored tremendous opportunities in parallel. The exponential growth in businesses, like online shopping, web designing, consumers trends, web usability, advertising, marketing, package design, and automotive engineering, through eye movements studies is sure and certain reality. On the one hand, the businesses have better chances of expansions based on innovative and promising findings of eye movement studies; on the other hand, eye movement studies have significant improvements and novel directions of researches as per business demands accordingly. Moreover, the businesses have liabilities to support the ongoing and future researches in eye movements, as these endeavors will have beneficial returns in businesses.

\section{Acknowledgements}

At first, I would like to thank the sponsor, Global IT Consultancy for Innovations, for this research program. Next, I 
am grateful to the dedicated participants for their appreciated efforts and precious time during experimentations. Further, I extend my gratitude and tribute to the great artist, Vincent Van Gogh for exceptional and marvelous pieces of art.

\section{References}

Ahmad, G. (2014). Analogical Thinking Induces Eye Movements in Scene Viewing. International Journal of Machine Learning and Computing, 4(1), 94-98. http://dx.doi.org/10.7763/IJMLC.2014.V4.393

Ahmad, G. (2014). Dynamics of Eye Gazing rely on Associative Relevance in Scene Viewing. Journal of Convergence Information Technology, 9(2), 35-42.

Ahmad, G. (2014). Flow of Analogical Thoughts controls Eye Movements in Scene Viewing. Journal of Next Generation Information Technology, 5(4), 118-125.

Ahmad, G. (2015). Associative Relevance Based Stimulus Shifts Focus in Eye Movements. International Business Research, 8(10), 25-34. http://dx.doi.org/10.5539/ibr.v8n10p25

Ahmad, G. (2015). Eye Fixation curves along Analogical Thinking in Scene Viewing. International Journal of Engineering and Industries, 6(1), 54-62.

Bly, B. M., \& Rumelhart, D. E. (Eds.). (1999). Cognitive Science. USA: Academic Press.

Bojko, A. (2013). Eye Tracking The User Experience: a practical guide to research. New York: Rosenfeld Media.

Brown, T. (1999). The Science and Art of Tracking. New York: NY: Berkley Books.

Duchowski, A. (2003). Eye Tracking Methodology: Theory and Practice. London: Springer Verlag.

Gentner, D. (1983). Structure-mapping: A theoretical framework for analogy. Cognitive Science, 7, 155-170. http://dx.doi.org/10.1207/s15516709 $\operatorname{cog} 0702 \_3$

Gentner, D., \& Markman, A. B. (1997). Structure-mapping in analogy and similarity. American Psychologist, 52, 45-56. http://dx.doi.org/10.1037/0003-066X.52.1.45

Gentner, D., \& Medina, J. (1998). Similarity and the development of rules. Cognition, 65, 263-297. http://dx.doi.org/10.1016/S0010-0277(98)00002-X

Gentner, D., Rattermann, M. J., \& Forbus, K. D. (1993). The roles of similarity in transfer: Separating retrievability from inferential soundness. Cognitive Psychology, 25, 524-575. http://dx.doi.org/10.1006/cogp.1993.1013

Gompel, R. P., Fischer, M. H., Murray, W. S., \& Hill, R. L. (Eds.). (2007). Eye Movements: A Window on Mind and Brain. UK: Elsevier.

Granka, L. A., Joachims, T., \& Gay, G. (2004). Eye-Tracking Analysis of User Behavior in WWW Search. SIGIR 04. Sheffield, South Yorkshire, UK: ACM. http://dx.doi.org/10.1145/1008992.1009079

Griffin, Z. M. (2004). Why look? Reasons for eye movements related to language production. In H. A. Ferreira (Ed.), The integration of language, vision, and action: Eye movements and the visual world (pp. 213-247). New York: Psychology Press.

Henderson, J. M., \& Hollingworth, A. (1998). Eye movements during Scene Viewing. An Overview. In G. W. Underwood (Ed.), Eye Guidance in Reading and Scene Perception (pp. 269-293). Oxford: Elsevier. http://dx.doi.org/10.1016/B978-008043361-5/50013-4

Henderson, J. M., \& Hollingworth, A. (1999). High-level Scene Perception. Annual Review of Psychology, 50(1), 243-271. http://dx.doi.org/10.1146/annurev.psych.50.1.243

Hermansen, O. (2015). Implimenting eye movements in business applications. Brage Bibsys, Hogskolen i Ostfold.

Hoffman, J. E. (1998). Visual Attention and Eye Movements. In H. Pashler (Ed.), Attention (pp. 119-153). London: Psychology Press.

Hofstadter, D., \& Sander, E. (2013). Surfaces and Essences: Analogy as the Fuel and Fire of Thinking. New York: Basic Books.

Holsanova, J. (2008). Discourse, Vision, and Cognition. New York: John Benjamins Publishing Company.

Koller, M., Salzberger, T., Brenner, G., \& Walla, P. (2012). Broadening the range of applications of eye-tracking in business research. Analise, Porto Alegre, 23(1), 71-77.

Liversedge, S. P., Gilchrist, I. D., \& Everling, S. (2011). The Oxford Handbook of Eye Movements. Oxford 
University Press. http://dx.doi.org/10.1093/oxfordhb/9780199539789.001.0001

Pan, B., Hembrooke, H. A., Gay, G. K., Granka, L. A., Feusner, M. K., \& Newman, J. K. (2004). The Determinants of Web Page Viewing Behavior: An Eye-Tracking Study. Association for Computing Machinery, 147-154. http://dx.doi.org/10.1145/968363.968391

Pan, B., Hembrooke, H., Joachims, T., Lorigo, L., Gay, G., \& Granka, L. (2007). In Google We Trust: Users' Decisions on Rank, Position, and Relevance. Journal of Computer-Mediated Communication, 12, 801-823. http://dx.doi.org/10.1111/j.1083-6101.2007.00351.x

Rayner, K. (1992). Eye movements and visual cognition: scene perception and reading. New York: Springer Verlag. http://dx.doi.org/10.1007/978-1-4612-2852-3

Snowden, R., Thompson, P., \& Troscianko, T. (2012). Basic Vision: An introduction to visual perception. Oxford University Press.

Solso, R. L. (1994). Cognition and Visual Arts. London: MIT Press.

Solso, R. L. (2003). The psychology of art and the evolution of the conscious brain. MIT Press.

Stark, L., \& Ellis, S. R. (1981). Scanpaths Revisited: Cognitive Models Direct Active Looking. In R. A. Dennis F. Fisher (Ed.), Eye Movements: Cognition and Visual Perception (pp. 193-226). Hillsdale: Lawrence Erlbaum Associates.

Tommasi, L., Peterson, M. A., \& Nadel, L. (Eds.) (2009). Cognitive Biology: Evolutionary and Developmental Perspectives on Mind, Brain and Behavior. London: MIT Press. http://dx.doi.org/10.7551/mitpress/9780262012935.001.0001

Tsotsos, J. K. (2011). A Computational Perspective on Visual Attention. London: MIT Press. http://dx.doi.org/10.7551/mitpress/9780262015417.001.0001

Turner, M. (2006). The Artful Mind - Cognitive Science and the riddle of human creativity. Oxford University Press.

Viegas, F. B., \& Wattenberg, M. (2007). Artistic Data Virtualization: Beyond Visual Analytics. Second International Conference on Online Communities and Social Computing, pp. 182-191.

Wells, A. (2000). Emotional Disorders and Metacognition: Innovative Cognitive Therapy. West Sussex: John Wiley \& Sons. http://dx.doi.org/10.1002/9780470713662 